\title{
Completud débil y Post completud en la escuela de Hilbert
}

\author{
Weak and Post completeness in the Hilbert school
}

\author{
Víctor Aranda \\ Universidad Autónoma de Madrid, España \\ victor.aranda@uam.es
}

\begin{abstract}
Resumen
El objetivo de este artículo es aclarar por qué la lógica proposicional es Post completa y su completud débil pasó casi desapercibida para Hilbert y Bernays, mientras que la lógica de primer orden es Post incompleta y su completud débil fue vista como un problema a resolver por Hilbert y Ackermann. Así, compararé la lógica proposicional y de primer orden en los Prinzipien der Mathematik, la segunda Habilitationsschrift de Bernays y los Grundzüge der Theoretischen Logik. La así llamada "interpretación aritmética", las formas normales conjuntiva y disyuntiva y la corrección de las reglas proposicionales de inferencia merecen especial énfasis.
\end{abstract}

Palabras clave: historia de la lógica, lógica clásica, formas normales, corrección, Bernays.

\begin{abstract}
The aim of this paper is to clarify why propositional logic is Post complete and its weak completeness was almost unnoticed by Hilbert and Bernays, while first-order logic is Post incomplete and its weak completeness was seen as an open problem by Hilbert and Ackermman. Thus, I will compare propositional and first-order logic in the Prinzipien der Mathematik, Bernays's second Habilitationsschrift and the Grundzüge der Theoretischen Logik. The so called "arithmetical interpretation", the conjunctive and disjunctive normal forms and the soundness of the propositional rules of inference deserve special emphasis.
\end{abstract}

Keywords: history of logic, classical logic, normal forms, soundness, Bernays.

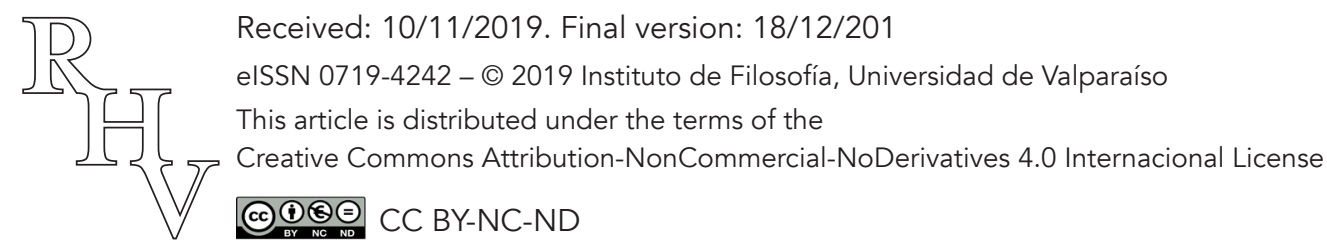




\section{Introducción}

En la literatura especializada ${ }^{1}$, hay un consenso general con respecto a que la primera vez que la completud (débil) de la lógica de primer orden se plantea como un problema a resolver es en Hilbert y Ackermann (1928). No obstante, los Grundzüge der Theoretischen Logik están basados en unas lecciones que Hilbert impartió en Gotinga, durante el semestre de invierno del curso 1917-18. En ellas, la cuestión de la completud de la lógica de primer orden ya está formulada ( $c f$. Hilbert 1917-18, 160). La pregunta, respondida afirmativamente por Gödel $(1929 ; 1930)$, es la siguiente: ¿son las fórmulas válidas de la lógica de primer orden también teoremas lógicos? (naturalmente, que todas las fórmulas válidas sean teoremas lógicos — esto es, fórmulas deducibles del cálculo sin premisassignifica que el conjunto de las mismas es recursivamente numerable). En palabras de Hilbert y Ackermann (1928):

Es todavía una cuestión sin resolver si el sistema de axiomas [de la lógica de primer orden] es completo al menos en el sentido de que todas las fórmulas lógicas que son verdaderas para todo dominio de individuos pueden ser deducidas a partir de los mismos ${ }^{2}$. (Hilbert y Ackermann 1928, 869)

El sistema de axiomas al que se refieren Hilbert y Ackermann es de sobra conocido. Se corresponde, además, con el fragmento de primer orden de los Principia Mathemati$c a$, aunque Bernays (1918) había mostrado que la proposición primitiva "Assoc" era en realidad deducible a partir de las demás ${ }^{3}$. Teniendo en cuenta que los condicionales son abreviaturas de fórmulas que contienen la negación y la disyunción, las conectivas primitivas en Hilbert y Ackermann (1928), los axiomas son los siguientes:

$$
\begin{array}{ll}
\text { 1. } & p \vee p \rightarrow p \\
\text { 2. } & p \rightarrow p \vee q \\
\text { 3. } & p \vee q \rightarrow q \vee p \\
\text { 4. } & (p \rightarrow q) \rightarrow[(r \vee p) \rightarrow(r \vee q)] \\
\text { 5. } & \forall x P(x) \rightarrow P(a) \\
\text { 6. } & P(a) \rightarrow \exists x P(x)
\end{array}
$$

Curiosamente, el hecho de que los axiomas 1-4 sean completos en el sentido especificado por Hilbert y Ackermann (o sea, el hecho de que la lógica proposicional es com-

\footnotetext{
${ }^{1} C f$., entre otros, Dreben y van Heijenoort (1986, 47), Zach (1999) y Mancosu $(2010,62)$.

2 "Ob das Axiomensystem wenigstens in dem Sinne vollständig ist, daß wirklich alle logischen Formeln, die für jeden Individuenbereich richtig sind, daraus abgeleitet werden können, ist eine noch ungelöste Frage" (Hilbert y Ackermann 1928, 869).

${ }^{3}$ Bernays informó a Russell de este resultado (y de la independencia del resto de axiomas) en una carta datada del 8 de abril de 1920 que puede consultarse en el archivo de Russell.
} 
pleta) no fue reivindicado por Hilbert - ni tampoco por Bernays - como un resultado de especial relevancia. Pues, en efecto, si el conjunto de fórmulas válidas de una lógica es decidible, entonces que sea recursivamente numerable no mejora nada. "In fact, from a computational point of view this property of decidability surpasses completeness" (Manzano y Alonso 2014, 2). Sin embargo, hay un segundo sentido en que los axiomas 1-4 son completos que sí ocupa un lugar destacado en Hilbert (1917-18) y Hilbert y Ackermann (1928). A este nuevo sentido de completud Hilbert lo llama "completud en sentido estricto" ("Vollständigkeit in dem schärferen Sinne").

La completud en sentido estricto se corresponde con lo que actualmente denominamos Post completud ${ }^{4}$. La demostración de que los axiomas 1-4 son Post completos explota el hecho de que existe un procedimiento efectivo para determinar si una fórmula es o no una tautología. En Hilbert (1917-18) y Hilbert y Ackermann (1928) ese procedimiento son las formas normales conjuntiva y disyuntiva. A partir de la Post completud (y de la corrección) de los axiomas 1-4, el corolario de que toda fórmula válida es una tautología es tan inmediato que pasó en buena medida desapercibido. Por el contrario, Ackermann demostró que los axiomas 1-6 (es decir, la lógica de primer orden) no son Post completos, dejando abierta la pregunta por su completud en el primer sentido.

El objetivo de este artículo es, precisamente, mostrar qué relación hay entre que la lógica proposicional sea Post completa y su completud sea inmediata y que la lógica de primer orden sea Post incompleta y su completud fuera vista como un problema a resolver. Para ello, he dividido el artículo en dos grandes bloques. Primero, se explican los conceptos de fórmula válida y de teorema lógico en la lógica proposicional que se desarrollaba en la escuela de Hilbert y su relación con la así llamada "interpretación aritmética" y las formas normales, conjuntiva y disyuntiva. Luego, se comenta la manera en que la Post completud y la corrección de los axiomas 1-4 implican que toda tautología es un teorema lógico, haciendo énfasis en el teorema de completud débil de Bernays. Finalmente, se introduce la "interpretación aritmética extendida", su relación con el problema de la consistencia de los axiomas 1-6 y la razón por la que estos axiomas son incompletos. Argumento que fue precisamente esta Post incompletud lo que permitió que la completud débil de la lógica de primer orden fuera vista como un problema abierto.

\footnotetext{
4 "Because an inconsistent system brings about the assertion of every proposition, Post defined a system to be inconsistent if it yields the assertion of the variable $p$ (which is equivalent to the derivability of every proposition if the substitution rule is present). From this notion derives our notion of Post completeness: A system of logic is Post complete if every time we add to it a sentence unprovable in it, we obtain an inconsistent system" (Mancosu 2010, 104).
}

Revista de Humanidades de Valparaíso, 2019, No 14, 449-466

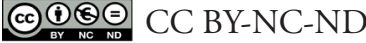




\section{Completud y Post completud en la lógica proposicional}

\subsection{La interpretación aritmética y las fórmulas válidas}

Hilbert, influido por la tradición comúnmente conocida como "Álgebra de la Lógica" sostenía que las expresiones del cálculo de enunciados ("Aussagenkalkül") pueden ser representadas como ecuaciones ( $c f$. Hilbert 1917-18, 107). Las expresiones que podemos igualar a 0 serán aquellas que, en tanto no dicen nada, son siempre verdaderas; las que podemos igualar a 1 serán las que son siempre falsas. Las primeras son, para Hilbert, las proposiciones lógicas, lo cual parece anticipar la concepción que Wittgenstein (1922) tenía de las mismas (véase, por ejemplo, el aforismo 4.463 del Tractatus). Después de haber introducido los axiomas 1-4, Hilbert construye la "interpretación aritmética" para mostrar que, efectivamente, dichos axiomas son proposiciones lógicas (esto es, que pueden ser igualados a 0 ).

La interpretación aritmética de los axiomas 1-4 toma los números 0 (para verdadero) y 1 (para falso) y sobre ellos se define la operación $1-p$, la suma y el producto lógicos, de tal modo que:

$$
\begin{array}{ll}
\text { - } & \neg p=1 \text { syss } p=0 ; 1-p \text { en otro caso. } \\
\text { - } & p \wedge q=1 \text { syss } p=1 \text { y } q=1 ; p+q \text { en otro caso. } \\
\text { - } & p \vee q=p q .
\end{array}
$$

Así pues, la negación de una fórmula es igual a 0 syss la fórmula es igual a 1 (ya que $1-1=0$ ), la conjunción de dos fórmulas es igual a 0 syss ambas fórmulas son iguales a 0 (puesto que $0+0=0$ ) y la disyunción de dos fórmulas es igual a 0 syss al menos una de ellas es igual a 0 (porque cualquier número multiplicado por 0 es igual a 0 ). Como es obvio, la manera en que están definidas estas operaciones coincide con la tabla de verdad de la negación, la conjunción y la disyunción, respectivamente. Si hablamos de la tabla de verdad de las conectivas es porque estas son operadores veritativo-funcionales. Es decir, el valor de verdad de las fórmulas que expresan negación, conjunción o disyunción depende únicamente del valor de verdad de sus constituyentes. Debido justamente a que son operadores veritativo-funcionales, Hilbert podía mostrar fácilmente que los axiomas 1-4 son proposiciones lógicas (o sea, fórmulas que pueden ser igualadas a 0 ).

Piensa, por ejemplo, en el primer axioma. Es evidente que, si $p=0$, entonces la fórmula es igual a 0 . Del mismo modo, si $p=1$, la fórmula también es igual a 0 . Por tanto, la fórmula puede ser igualada a 0 independientemente de si $p$ es verdadera o falsa (de ahí que sea una proposición lógica). En símbolos:

\footnotetext{
5 "Equating two relative expressions yields a relative equation, which we shall always think of as brought into zero form. All important problems of mathematics and of the calculus of logic can, it seems, be reduced to [questions about] such relative equations" (Löwenheim 1915, 233).
} 
Completud débil y Post completud en la escuela de Hilbert

Víctor Aranda

- $\quad p \vee p \rightarrow p=\neg(p \vee p) \vee p=[1-(00)] 0=1 \quad 0=0$

- $\quad p \vee p \rightarrow p=\neg(p \vee p) \vee p=[1-(11)] 1=0 \quad 1=0$

La demostración de que los axiomas 2-4 son fórmulas válidas es igualmente simple. De hecho, Hilbert (1917-18, 154) omite la prueba afirmando que es algo que puede "verificarse directamente". Hilbert y Ackermann (1928, 836-37), en cambio, sí comprueban uno a uno que los axiomas 1-4 son proposiciones lógicas. Además, esta "interpretación aritmética" permite a Bernays (1918) introducir el concepto de "fórmula válida" ("allgemeingültigen Formel') en términos prácticamente contemporáneos:

Se entiende por fórmula 'deducible' aquella que puede ser demostrada como una fórmula correcta a partir de los axiomas y por fórmula 'válida' aquella que, en el sentido de la interpretación dada, cualquier elección de enunciados para las variables (es decir, para cualquier 'valor' de las variables) siempre resulta en un enunciado verdadero'. (Bernays 1918, 236)

Es importante destacar que, en este contexto, el término "interpretación" tiene un significado distinto al habitual. Normalmente, decimos que una fórmula es válida syss es verdadera en toda interpretación, donde una interpretación es un par formado por una estructura y una función de asignación. Si no es válida, habrá al menos una interpretación donde es falsa. Sin embargo, Bernays entiende por "interpretación" la interpretación aritmética antes mencionada. Y, en el fondo, la interpretación aritmética es lo que fija el significado de las constantes lógicas (en este caso, de las conectivas). Esto es, independientemente de $p, q, r \ldots$, la negación, la conjunción y la disyunción son las funciones definidas más arriba. Mientras que en lógica contemporánea la verdad de una fórmula se evalúa en diferentes interpretaciones, la interpretación aritmética mantiene fijo el significado de las conectivas, se evalúe una fórmula u otra. Lo único que varía es, pues, la asignación de las letras proposicionales.

Por tanto, para Bernays una fórmula válida es aquella que resulta en una proposición verdadera para cada posible valor de sus letras proposicionales. Así, cuando afirma que una fórmula es verdadera "bajo la interpretación aritmética" no hay que entender que es verdadera "en un modelo", sino más bien que es igual a 0 cuando el significado de las constantes lógicas está determinado por lo que hoy consideramos la semántica estándar de las conectivas. Y, al tratarse de operadores veritativo-funcionales, la validez o invalidez de una fórmula puede determinarse a través de un procedimiento efectivo.

\footnotetext{
6 "Versteht man unter einer 'beweisbaren' Formel eine solche, die sich gemäss den Axiomen als richtige Formel erweisen lässt, und unter einer 'allgemeingültigen' Formel eine solche, die im Sinne der angegebenen Deutung bei beliebiger Wahl der für die Variablen einzusetzenden Aussagen (also für beliebige 'Werte' der Variablen) stets eine wahre Aussage ergibt" (Bernays 1918, 236).
} 
Completud débil y Post completud en la escuela de Hilbert

Víctor Aranda

\subsection{Las formas normales y los teoremas lógicos}

En los Grundzüge der Theoretischen Logik, los teoremas lógicos son aquellas fórmulas proposicionales que son "deducibles" a partir de los axiomas 1-4. Sin embargo, en su definición de teorema lógico no hablan de las reglas del cálculo, sino de la forma normal conjuntiva:

Una fórmula proposicional es deducible a partir de los axiomas si y solo si, en la correspondiente forma normal conjuntiva, cada sumando contiene dos factores, cada uno de los cuales es el contrario del otro ${ }^{7}$. (Hilbert y Ackermann 1928, 835)

Una fórmula está en forma normal conjuntiva (en adelante, FNC) syss es una conjunción de cláusulas, cada una de las cuales es una disyunción de literales. Las cláusulas son los "sumandos" de la conjunción, mientras que los literales son los "factores" de la disyunción. La razón por la que una fórmula en FNC cuyas cláusulas siempre contengan literales mutuamente contradictorios puede ser igualada a 0 (o sea, es una proposición lógica) es bastante obvia. Expresado en términos de la interpretación aritmética, cada cláusula contendrá al menos un 0 , pues $p \vee \neg p=0$. Puesto que cualquier número multiplicado por 0 es 0 , el valor de las cláusulas siempre será 0 , obteniendo así una conjunción de ceros, esto es, $0 \wedge 0 \wedge 0 \wedge \ldots \wedge 0$. Dado que la conjunción se interpreta como la suma cuando toma como argumentos 0,0 , es evidente que el valor de la conjunción de ceros será siempre 0. Por tanto, toda fórmula en FNC cuyas cláusulas siempre tienen literales contradictorios es igual a 0 , independientemente de la asignación de valores de verdad a dichos literales.

Según Hilbert y Ackermann $(1928,820)$, la tarea de la lógica es encontrar qué fórmulas son proposiciones lógicas ${ }^{8}$. Esta tarea se puede reducir a la de decidir si en todo producto de una fórmula en FNC aparecen, como mínimo, dos literales contradictorios como factores, porque toda fórmula es equivalente a una fórmula en FNC. Las reglas para la transformación de una fórmula en su FNC son las siguientes ( $c f$. Hilbert y Ackermann 1928, 818):

- La asociatividad, la conmutatividad y la distributividad de la conjunción y de la disyunción.

- $\quad$ La equivalencia entre $\neg \neg p \mathrm{y} p$.

\footnotetext{
7 "Denn eine Aussagenformel ist dann und nur dann aus den Axiomen beweisbar, wenn in der zugehörigen konjunktiven Normalform jeder Summand zwei Faktoren enthält, von denen der eine das Gegenteil des anderen ist" (Hilbert y Ackermann 1928, 835).

8 "La gran cuestión ahora”, reflexionaba Wittgenstein en una carta a Russell, "es: ¿cómo debe construirse un sistema de signos a fin de hacer que toda tautología sea reconocible como tal DE UNA SOLA Y ÚNICA MANERA? ¡Este es el problema fundamental de la lógica!” (Monk 1994, 103)
}

Revista de Humanidades de Valparaíso, 2019, No 14, 449-466

(c) $(1) \Theta($ ) CC BY-NC-ND 
- $\quad$ La equivalencia ${ }^{9}$ entre $\neg(p \wedge q) \mathrm{y} \neg p \vee \neg q$ y la equivalencia entre $\neg(p \vee q) \mathrm{y}$ $\neg p \wedge \neg q$.

- $\quad$ Que $p \rightarrow q$ puede escribirse como $\neg p \vee q$ y que $p \leftrightarrow q$ puede escribirse como $\neg p \vee q \wedge p \vee \neg q$.

Por otro lado, Hilbert y Ackermann $(1928,822)$ también introducen la forma normal disyuntiva (en adelante, FND) para identificar qué fórmulas son iguales a 1 con independencia de la asignación de valores a los enunciados primitivos (las reglas para la transformación de una fórmula en esta segunda forma normal son las mismas):

Al igual que se podía inferir de una forma normal conjuntiva si una expresión era siempre verdadera o no lo era, también se puede decidir con ayuda de la disyuntiva si es siempre falsa o no lo es. Lo es si y solo si cada factor contiene simultáneamente un enunciado primitivo y su negación como sumandos ${ }^{10}$. (Hilbert y Ackermann 1928, 822)

Una fórmula está en FND syss es una disyunción de cláusulas, cada una de las cuales es una conjunción de literales. Las cláusulas son los "factores" de la disyunción, mientras los literales son los "sumandos" de la conjunción. La razón por la que una fórmula en FND cuyas cláusulas siempre contengan literales mutuamente contradictorios puede ser igualada a 1 (o sea, es una contradicción) es, de nuevo, bastante obvia. Expresado en términos de la interpretación aritmética, cada cláusula contendrá al menos un 1, porque $p \wedge \neg p=1$. Dado que cualquier número sumado a 1 es 1 (intuitivamente, cualquier conjunción con un "sumando" falso será falsa), el valor de las cláusulas siempre será 1, obteniendo así una disyunción de unos, esto es, $1 \vee 1 \vee 1 \vee \ldots \vee 1$. Y, como la disyunción se interpreta como la multiplicación cuando toma como argumentos 1,1 , es evidente que el valor de la disyunción de unos será siempre 1. Por tanto, toda fórmula en FND cuyas cláusulas siempre tienen literales contradictorios es igual a 1, independientemente de la asignación de valores de verdad a dichos literales.

Hilbert y Ackermann $(1928,825)$ afirman que la solución a los problemas de validez y satisfacibilidad para el cálculo de enunciados pasan por la transformación de las fórmulas a FNC y FND, respectivamente. El problema de la validez consiste en decidir, mediante un procedimiento finito, si una expresión del cálculo de enunciados es siempre verdadera o no lo es; el de la satisfacibilidad, si hay una asignación de valores que la hace verdadera. Una fórmula $\varphi$ es válida si su equivalente en FNC es una conjunción de ceros; es satisfa-

\footnotetext{
${ }^{9}$ Naturalmente, estas equivalencias son las leyes de De Morgan para la conjunción y la disyunción, respectivamente.

10 "Wie man aus der konjunktiven Normalform ablesen kann, ob ein Ausdruck immer richtig ist oder nicht, so kann man mit Hilfe der disjunktiven entscheiden, ob er immer falsch ist. Dies ist dann und nur dann der Fall, wenn jeder Faktor mit einer Grundaussage zugleich ihr Gegenteil als Summand enthält". (Hilbert y Ackermann 1928, 822).
}

Revista de Humanidades de Valparaiso, 2019, No 14, 449-466 
cible si su equivalente en FND no es una conjunción de unos. Adviértase que la definición de teorema lógico ( $c f$. Hilbert y Ackermann 1928, 835) coincide exactamente con el criterio para determinar si una fórmula es válida. Pues, en efecto, si el equivalente de $\varphi$ en FNC es una conjunción de ceros es porque cada cláusula (cada "sumando") contiene al menos dos literales mutuamente contradictorios como factores.

Finalmente, en la décima sección del capítulo dedicado a la lógica proposicional, Hilbert y Ackermann $(1928,829)$ introducen la distinción entre "axiomas formales" y "axiomas materiales". Los axiomas formales son los axiomas 1-4, mientras que los axiomas materiales son las reglas primitivas para la deducción de fórmulas verdaderas. Estas reglas son las siguientes ( $c f$. Hilbert y Ackermann 1928, 830):

- Regla de sustitución ${ }^{11}$ : una letra sentencial puede ser sustituida, donde quiera que ocurra, por una y el mismo enunciado compuesto.

- $\quad$ Esquema de deducción (modus ponens): a partir de las fórmulas $\alpha \rightarrow \beta$ y $\alpha$, se obtiene la nueva fórmula $\beta$.

En mi opinión, la razón de que estas reglas de inferencia se presenten tan tarde en la discusión es que existe un procedimiento efectivo para determinar si una fórmula es o no una proposición lógica, así que no hace falta que nos preocupemos por encontrar una prueba de que la fórmula válida en cuestión se sigue de los axiomas (pues ya sabemos que lo hace). Por tanto, y como señalaba el propio Bernays, "el cálculo puede trivializarse completamente" (Bernays 1918, 241).

Después de derivar reglas de deducción adicionales y poner algunos ejemplos de deducción de fórmulas a partir de las mismas ( $c f$. Hilbert y Ackermann 1928, 831-35), en los apartados finales de este capítulo de los Grundzüge se abordan las cuestiones metalógicas del cálculo de enunciados.

\subsection{El teorema de completud débil en Bernays}

En su segunda tesis de habilitación, Bernays (1918) formula los teoremas de corrección y completud débil para la lógica proposicional de una manera muy precisa:

Se entiende por fórmula 'deducible' aquella que puede ser demostrada como una fórmula correcta a partir de los axiomas y por fórmula 'válida' aquella que, en el sentido de la interpretación dada, cualquier elección de enunciados para las variables (es decir, para cualquier

\footnotetext{
${ }^{11}$ Aunque en los Principia Mathematica se usa profusamente la regla de sustitución, no se hace explícita. Bernays (1918) y Lewis (1918) sí la incluyen como una regla de inferencia independiente.
} 
'valor' de las variables) siempre resulta en un enunciado verdadero, por lo que se tiene el teorema: toda fórmula deducible es una fórmula válida y viceversa ${ }^{12}$. (Bernays 1918, 236)

La conversa del teorema es la completud débil. La demostración de completud débil es inmediata a partir de la corrección y la Post completud - es esencialmente la misma en Hilbert (1917-18) y Bernays (1918) - . Por esta razón, lo primero que se prueba es que la regla de sustitución y el esquema de deducción preservan la validez de los axiomas 1-4. Considera, por ejemplo, el primero de ellos. En virtud de la regla de sustitución, podemos sustituir uniformemente la letra sentencial $p$ por uno y el mismo enunciado compuesto. Es evidente que, si el enunciado compuesto es falso, entonces la fórmula resultante es igual a 0 y que, si es verdadero, también lo será. De este modo, cualquier expresión proposicional que resulte de aplicar la regla de sustitución en 1 es una proposición lógica (y, por tanto, una fórmula válida). Como vimos más arriba, estas son las dos posibilidades de sustitución:

$$
\begin{aligned}
& \text { - } \quad p \vee p \rightarrow p=\neg(p \vee p) \vee p=[1-(00)] \quad 0=1 \quad 0=0 \\
& \text { - } \quad p \vee p \rightarrow p=\neg(p \vee p) \vee p=[1-(11)] 1=0 \quad 1=0
\end{aligned}
$$

La prueba es obvia, pero tediosa, para el resto de axiomas 2-4. La demostración de que el modus ponens también preserva la validez es igualmente sencilla. Consiste en mostrar que, si deducimos la fórmula $\beta$ a partir de dos fórmulas $\alpha \rightarrow \beta$ y $\alpha$ que son siempre iguales a 0 , entonces $\beta$ también debe ser siempre igual a 0 . Que $\alpha \rightarrow \beta$ sea siempre igual a 0 significa que $\neg \alpha \vee \beta=0$ independientemente del valor asignado a los constituyentes de $\alpha$ y $\beta$. Por el supuesto inicial, sabemos que $\alpha$ es siempre igual a 0 , por lo que $\neg \alpha$ es siempre igual a 1 (esto es, es una contradicción). Por tanto, para que $\neg \alpha \vee \beta$ sea siempre igual a $0, \beta$ debe ser una fórmula válida. De lo contrario, $\alpha \rightarrow \beta$ no sería siempre igual a 0 , lo cual contradice el supuesto inicial. Y, como $\beta$ debe ser siempre igual a 0 , el esquema de deducción preserva la propiedad ${ }^{13}$ de ser siempre igual a 0 , o sea, la validez ( $c f$. Hilbert y Ackermann 1928, 837).

De la corrección de los axiomas materiales se sigue la consistencia de los axiomas del cálculo de enunciados. Para Hilbert y Ackermann (1928, 836), estos axiomas serán consistentes (en el sentido de "libres de contradicción") si es imposible deducir a partir de ellos, con ayuda del cálculo, dos enunciados $p, \neg p$. Naturalmente, si sabemos que

\footnotetext{
12 "Versteht man unter einer 'beweisbaren' Formel eine solche, die sich gemäss den Axiomen als richtige Formel erweisen lässt, und unter einer 'allgemeingültigen' Formel eine solche, die im Sinne der angegebenen Deutung bei beliebiger Wahl der für die Variablen einzusetzenden Aussagen (also für beliebige 'Werte' der Variablen) stets eine wahre Aussage ergibt, so gilt der Satz: Jede beweisbare Formel ist eine allgemeingültige Formel und umgekehrt"' (Bernays 1918, 236).

${ }^{13} \mathrm{La}$ idea que subyace a esta prueba de consistencia es la misma que está en su primer prototipo de prueba de consistencia y en sus "ecuaciones homogéneas" ( $c f$. Hilbert 1904, 134).
} 
dichos axiomas son válidos y que las reglas de inferencia preservan la validez, entonces toda fórmula que se deduzca de ellos es siempre igual a 0 . Puesto que la fórmula $p \wedge \neg p$ es siempre igual a 1, una contradicción nunca podrá seguirse de los axiomas 1-4. En consecuencia, son consistentes.

Una vez probada la corrección, el segundo paso para establecer la completud débil de la lógica proposicional es demostrar la Post completud de los axiomas 1-4. Que sean Post completos significa que la adición de una fórmula que no sea deducible a partir de los mismos hace que se vuelvan inconsistentes. Desde un punto de vista contemporáneo, esta afirmación resulta bastante extraña, porque no es cierto que la adición de, por ejemplo, el literal $p$, haga contradictorio el conjunto de los axiomas 1-4. Esto implicaría que $\neg p$ es un teorema lógico (lo cual es falso) y que el conjunto de los axiomas 1-4 es máximamente consistente, o sea, que la teoría de la lógica proposicional es completa (lo cual es, nuevamente, falso). ¿En qué sentido podemos decir, pues, que la lógica proposicional Post completa?

Ten en cuenta que, para Hilbert (1917-18) y Hilbert y Ackermann (1928), una fórmula proposicional es deducible a partir de los axiomas 1-4 syss su correspondiente FNC es una conjunción de ceros. De ahí que una fórmula que no lo sea es equivalente a una expresión en FNC que no es una conjunción de ceros: al menos una de sus cláusulas no es siempre igual a 0. Así, la demostración de Post completud parte de una fórmula $\varphi$ cuya FNC (sea esta $\Psi$ ) contiene al menos una cláusula que no es siempre igual a 0 . Supongamos que añadimos $\varphi$ a los axiomas 1-4. Sabemos, pues, que $\Psi$ tiene un "sumando", una cláusula (sea esta $\mathrm{C}$ ), que no tiene literales mutuamente contradictorios como factores. Por ello, si sustituimos en $\mathrm{C}$ todos los literales afirmados por el enunciado $p$ y todos los negados por $\neg p$, entonces $\mathrm{C}$ es de la forma $p \vee p \vee p \vee \ldots \vee p$, que es equivalente a $p$. Sin embargo, también podríamos sustituir ahora $p$ por el enunciado $\neg p$, de tal manera que $\mathrm{C}$ fuera de la forma $\neg p \vee \neg p \vee \neg p \vee \ldots \vee \neg p$, que es equivalente a $\neg p$. Por tanto, a partir de la regla de sustitución y de la adición de una fórmula no deducible a los axiomas 1-4, obtenemos una contradicción (cf. Hilbert y Ackermann 1928, 839).

Bernays $(1918,238)$, en cambio, modifica ligeramente la definición de Hilbert para que sea independiente de la negación: los axiomas 1-4 son Post completos, porque la adición al conjunto de una fórmula no deducible a partir de los mismos haría que cualquier fórmula lo fuera ${ }^{14}$. No obstante, es evidente que son equivalentes: de una contradicción, se sigue cualquier cosa.

La clave está en entender que, si añadimos una fórmula no deducible al conjunto de axiomas 1-4, el conjunto resultante deja de ser un conjunto de fórmulas iguales a 0 en la

14 “Dieser Satz steht in engem Zusammenhange mit der Frage der Vollständigkeit unseres Axiomen Systems. Wir brauchen nämlich zu seinem Nachweise nur zu zeigen, dass es unmöglich ist, ausser den beweisbaren Formeln noch einen Ausdruck des Kalküls als richtige Formel zu postulieren, ohne dass sämtliche Ausdrücke sich als richtige Formeln ergeben" (Bernays 1918, 238).

Revista de Humanidades de Valparaíso, 2019, No 14, 449-466 
interpretación aritmética. Esta es la intuición que explota Bernays (1918) en su prueba de completud débil para el cálculo de enunciados. Así, razona Bernays (1918, 238), si hubiera una fórmula válida que no fuera deducible a partir de los axiomas 1-4 podríamos añadirla como el axioma número $5^{*}$. Por la Post completud de los axiomas 1-4, esto implicaría que cualquier fórmula es deducible a partir del nuevo conjunto de axiomas $1-5^{*}$. Y, por la corrección de la regla de sustitución y del esquema de deducción, de ahí se concluiría que cualquier fórmula es válida, lo cual es obviamente falso. Luego no hay ninguna fórmula válida que no sea deducible a partir de los axiomas 1-4, esto es, toda fórmula válida es un teorema lógico.

Con esto termina la demostración de Bernays del teorema de completud débil.

\section{Completud y Post incompletud en la lógica de primer orden}

\subsection{Extendiendo la interpretación aritmética}

En la novena sección del capítulo dedicado a lo que hoy llamamos "lógica de primer orden", Hilbert y Ackermann $(1928,866)$ se preguntan por la consistencia y la completud de los axiomas 1-6. El axioma 5 decía que, "si el predicado $P$ se aplica a todos $\operatorname{los} x$, entonces se aplica a un $a$ particular"; el axioma 6, que "si el predicado $P$ se aplica a un $a$ particular, entonces hay un $x$ al que se aplica" ( $c f$. Hilbert y Ackermann 1928, 856). Ellos argumentan que el método de la interpretación aritmética, que permitía probar la consistencia de los axiomas 1-4, también es útil para abordar el problema de la consistencia de los axiomas 1-6.

La interpretación aritmética para los axiomas 1-6 mantiene el significado estándar de las conectivas. Solo son posibles los valores de verdad 0 y 1 , de tal manera que $\neg 0=1 \mathrm{y}$ $\neg 1=0$. Es fundamental destacar que el universo de esta interpretación es un único elemento, por lo que la prueba de consistencia no debe sobreestimarse:

La importancia del resultado de esta prueba de consistencia para nuestros axiomas no se debería sobreestimar. El contenido de la demostración dada se reduce al presupuesto de que el dominio de individuos en el que se basa consta solo de un único elemento ${ }^{15}$. (Hilbert y Ackermann 1928, 867)

Es fácil mostrar que, de acuerdo con la interpretación aritmética extendida, los axiomas 5-6 son siempre iguales a 0. Vayamos con el axioma número 5. Supongamos que el predicado $P$ se aplica al único elemento del universo, sea este $a$. Es evidente que, en tal

\footnotetext{
15 "Man darf das Ergebnis dieses Beweises für die Widerspruchsfreiheit unserer Axiome übrigens in seiner Bedeutung nicht überschätzen. Der angegebene Beweis der Widerspruchsfreiheit kommt nämlich inhaltlich darauf hinaus, daß man annimmt, der zugrunde gelegte Individuenbereich bestehe nur aus einem einzigen Element" (Hilbert y Ackermann 1928, 867).
}

Revista de Humanidades de Valparaíso, 2019, No 14, 449-466 
caso, la fórmula $\forall x P(x) \rightarrow P(a)=0$. Supongamos, por el contrario, que el predicado $P$ no se aplica al único elemento $a$ del universo. Puesto que la fórmula $\forall x P(x) \rightarrow P(a)$ es equivalente a $\neg \exists x P(x) \vee P(a)$ y es cierto que $\neg \exists x P(x)=0$ (ya que $a$, el único elemento del universo, no tiene la propiedad $P$ ), es evidente que $\forall x P(x) \rightarrow P(a)=0$. Por tanto, el axioma número 5 es siempre igual a 0 bajo la interpretación aritmética extendida. Pasemos ahora al axioma número 6. Supongamos que el predicado $P$ se aplica al único elemento del universo. En tal caso, es obvio que $P(a) \rightarrow \exists x P(x)=0$. Supongamos, por el contrario, que no se aplica. Dado que la fórmula $P(a) \rightarrow \exists x P(x)$ es equivalente a $\neg P(a) \vee \exists x P(x)$ y es cierto que $\neg P(a)=0$ (pues $a$ no tiene la propiedad $P$ ), se tiene que $P(a) \rightarrow \exists x P(x)=0$. En consecuencia, el axioma número 6 es siempre igual a 0 bajo la interpretación aritmética extendida.

Ahora bien, ¿son los axiomas 5-6 proposiciones lógicas (o sea, fórmulas válidas)? En el cálculo de enunciados, una proposición lógica es toda fórmula proposicional cuya FNC es una conjunción de ceros. Hilbert y Ackermann $(1928,868)$ reducen la validez de los axiomas 5-6 a la validez de la fórmula que resulta de transformar $\forall x P(x) \rightarrow P(a)$ y $P(a)$ $\rightarrow \exists x P(x)$ en expresiones proposicionales. El primer paso es definir una nueva interpretación para los mismos cuyo universo conste de dos elementos, sean estos $a$ y $b$. El segundo paso es sustituir las sentencias universales por la conjunción de sus dos instancias de cuantificación y las sentencias existenciales por la disyunción de sus dos instancias de cuantificación. De este modo, $\forall x \Phi(x)$ es equivalente a $\Phi(a) \wedge \Phi(b)$ y $\exists x P(x)$ es equivalente a $\Phi(a) \vee \Phi(b)$. El tercer paso es cuantificar universalmente tanto $\forall x P(x) \rightarrow P(a)$ como $P(a) \rightarrow \exists x P(x)$, de tal manera que pasamos a $\forall y(\forall x P(x) \rightarrow P(y))$ y $\forall y(P(y) \rightarrow \exists x P(x))$.

Centrándonos en el axioma 5, a partir de la fórmula $\forall y(\forall x P(x) \rightarrow P(y))$ obtenemos la conjunción $\forall x P(x) \rightarrow P(a) \wedge \forall x P(x) \rightarrow P(b)$. De ahí se sigue que $(P(a) \wedge P(b) \rightarrow P(a))$ $\wedge(P(a) \wedge P(b) \rightarrow P(b))$, lo cual es equivalente a la expresión proposicional $(p \wedge q \rightarrow p)$ $\wedge(p \wedge q \rightarrow q)$, es decir, a $(\neg(p \wedge q) \vee p) \wedge(\neg(p \wedge q) \vee q)$. Por la ley de De Morgan para la conjunción, esto equivale a $(\neg p \vee \neg q \vee p) \wedge(\neg p \vee \neg q \vee q)$. En la primera cláusula (en el primer "sumando"), $\neg p$ y $p$ son literales mutuamente contradictorios; en el segundo "sumando", lo son $\neg q$ y $q$. Por tanto, la FNC correspondiente es $0^{\wedge} 0$, esto es, el axioma 5 es una proposición lógica. En lo que respecta al axioma 6 , a partir de la fórmula $\forall y(P(y)$ $\rightarrow \exists x P(x)$ ) obtenemos la conjunción $P(a) \rightarrow \exists x P(x) \wedge P(b) \rightarrow \exists x P(x)$. De ahí se sigue que $(P(a) \rightarrow P(a) \vee P(a)) \wedge(P(b) \rightarrow P(a) \vee P(a))$, lo cual es equivalente a la expresión proposicional $(p \rightarrow p \vee q) \wedge(q \rightarrow p \vee q)$ o, lo que es lo mismo, a $(\neg p \vee p \vee q) \wedge(\neg q \vee p$ $\vee q$ ). Vemos que en el primer sumando $\neg p$ y $p$ aparecen como factores; en el segundo, lo hacen $\neg q$ y $q$. En consecuencia, la FNC correspondiente es $0^{\wedge} 0$, así que el axioma 6 es una proposición lógica.

Esto completa la prueba de que los axiomas 5-6 son proposiciones lógicas. La siguiente pregunta que se hacen Hilbert y Ackermann $(1928,867)$ es si son Post completos (que ellos llaman "completud en sentido estricto"). Es decir, si la adición de una fórmula no deducible a partir de los axiomas 1-6 hace que el conjunto de fórmulas resultante deje de 
ser un conjunto de fórmulas iguales a 0 en la interpretación aritmética extendida. $\mathrm{O}$, en otras palabras, si no hay ninguna fórmula que no sea deducible a partir de los axiomas de la lógica de primer orden y que sea igual a 0 en dicha interpretación.

\subsection{La Post incompletud de la lógica de primer orden}

Como una fórmula se considera deducible a partir de los axiomas lógicos si su FNC es una conjunción de ceros, el problema se reduce a averiguar si hay alguna fórmula de primer orden equivalente a una FNC que no es una conjunción de ceros (que no es, pues, siempre verdadera) y que, en cambio, sea igual a 0 en la interpretación aritmética extendida. En Hilbert y Ackermann $(1928,867)$, se demuestra que esa fórmula existe y que, debido a ello, los axiomas 1-6 no son Post completos:

Para determinar la incompletud del sistema de axiomas, solo necesitamos encontrar una fórmula que, de acuerdo con la interpretación aritmética de más arriba, sea igual a 0 , pero no una consecuencia de los axiomas ${ }^{16}$. (Hilbert y Ackermann 1928, 867)

En una nota al pie, el crédito de la prueba se atribuye a Ackermann. La fórmula es la siguiente:

$$
\delta:=\exists x P(x) \rightarrow \forall x P(x)
$$

El primer paso de la demostración consiste en mostrar que la fórmula es igual a 0 en la interpretación aritmética extendida. Recordemos que el universo de la interpretación aritmética consta de un único elemento, sea este $a$. Supongamos que el predicado $P$ se aplica al elemento $a$. En tal caso, es evidente que la fórmula $\exists x P(x) \rightarrow \forall x P(x)=0$, porque su consecuente, $\forall x P(x)$, es verdadero ( $a$ es el único elemento del universo). Supongamos, por el contrario, que el predicado $P$ no se aplica al elemento $a$. Puesto que la fórmula $\exists x P(x) \rightarrow \forall x P(x)$ es equivalente a $\neg \exists x P(x) \vee \forall x P(x)$, es obvio que $\neg \exists x P(x)=0$ (porque el único elemento del dominio no tiene la propiedad $P$ ), así que $\exists x P(x) \rightarrow \forall x P(x)=0$. De ahí que la fórmula $\exists x P(x) \rightarrow \forall x P(x)$ sea siempre igual a 0 en la interpretación aritmética extendida.

Sin embargo, el segundo paso de la prueba muestra que $\exists x P(x) \rightarrow \forall x P(x)$ no es deducible a partir de los axiomas 1-6. Según Hilbert y Ackermann $(1928,867)$, esta fórmula puede sustituirse por $(P(a) \vee P(b)) \rightarrow(P(a) \wedge P(b))$, lo cual es equivalente a la expresión proposicional $(p \vee q) \rightarrow(p \wedge q)$ o, lo que es lo mismo, $\neg(p \vee q) \vee(p \wedge q)$. Por la ley de De Morgan para la disyunción, esto equivale a $(\neg p \wedge \neg q) \vee(p \wedge q)$. Por la propiedad dis-

16 “Um die Unvollständigkeit des Axiomensystems festzustellen, brauchen wir nur eine Formel zu finden, welche gemäß der obigen arithmetischen Deutung identisch gleich 0, aber nicht eine Konsequenz der Axiome ist" (Hilbert y Ackermann 1928, 867). 
Completud débil y Post completud en la escuela de Hilbert

Víctor Aranda

tributiva de la conjunción y la disyunción, obtenemos la fórmula $(\neg p \vee p) \wedge(\neg p \vee q) \wedge$ $(\neg q \vee p) \wedge(\neg q \vee q)$. Como se advierte, solo el primer y el último sumando tienen literales mutuamente contradictorios como factores, por lo que no se trata de una conjunción de ceros. Así pues, $\delta$ no es una fórmula deducible a partir de los axiomas 1-6 (no es, en definitiva, una proposición lógica).

Esto implica, pues, que si añadiésemos $\delta$ al conjunto de axiomas 1-6, el conjunto resultante seguiría siendo un conjunto de fórmulas iguales a 0 en la interpretación aritmética extendida, por lo que dichos axiomas no son Post completos. En otras palabras, implica que hay fórmulas que no son deducibles a partir de los axiomas 1-6, pero que son verdaderos en la interpretación aritmética extendida. ¿Singnifica esto que hay fórmulas válidas que no son deducibles a partir de los axiomas 1-6 (esto es, que no son teoremas lógicos)? Evidentemente, no. Ackermann no probó que la lógica de primer orden es incompleta. De hecho, sabemos por Gödel $(1929 ; 1930)$ que sí que lo es: toda fórmula válida de esta lógica es un teorema lógico. Lo que podemos concluir del resultado de Ackermann es que, en primer orden, ser siempre igual a 0 en la interpretación aritmética extendida no es equivalente a ser válido. Y esto permite entender por qué la completud (débil) de la lógica de primer orden era vista como un problema a resolver en los Grundzüge der Theoretischen Logik.

En la lógica proposicional, ser siempre igual a 0 en la interpretación aritmética es equivalente a ser válido. Esto se debe, en realidad, a que la validez en esta lógica es decidible, esto es, puede determinarse con arreglo a un procedimiento finito (las formas normales conjuntivas). Las conectivas son operadores veritativo-funcionales. Por tanto, probar que toda expresión proposicional que es igual a 0 en la interpretación aritmética es lógicamente deducible a partir de los axiomas 1-4 es demostrar la completud débil del fragmento proposicional. Sin embargo, ser siempre igual a 0 en la interpretación aritmética extendida no es equivalente a ser válido. Si una fórmula es siempre igual a 0 en la interpretación aritmética extendida, entonces es verdadera en una interpretación cuyo universo tiene un único elemento. No obstante, una fórmula como $\delta$, que es verdadera en la interpretación aritmética extendida, es falsa en ciertas interpretaciones con más de un elemento (piensa, por ejemplo, en una cuyo universo contenga dos elementos y solo uno de ellos tenga la propiedad $P$ ).

Así pues, una vez que la Post incompletud de los axiomas 1-6 ha puesto de manifiesto que no todas las fórmulas iguales a 0 en la interpretación aritmética extendida son deducibles a partir de los mismos, la siguiente pregunta aflora con naturalidad: ¿son las fórmulas iguales a 0 en toda interpretación (y no solo en la aritmética extendida) deducibles a partir de los axiomas 1-6? Es decir, ¿es la lógica de primer orden débilmente completa?

Es todavía una cuestión sin resolver si el sistema de axiomas [de la lógica de primer orden] es completo al menos en el sentido de que todas las fórmulas lógicas que son verdaderas

Revista de Humanidades de Valparaíso, 2019, No 14, 449-466

(c) $(1)(9)$ CC BY-NC-ND 
Completud débil y Post completud en la escuela de Hilbert

Víctor Aranda

para todo dominio de individuos pueden ser deducidas a partir de los mismos ${ }^{17 "}$ (Hilbert y Ackermann 1928, 869).

\subsection{Breve reflexión final: protosemántica y completud}

En un artículo relativamente reciente, Manzano y Alonso (2014) señalan que el uso de formas normales y de tablas de verdad a finales de la década de 1910 y principios de 1920 - piensa no solo en Bernays (1918) y Hilbert (1917-18), sino también en Post (1921) o en Wittgenstein (1922) - podría hacernos creer que había un entendimiento lo suficientemente refinado de las nociones semánticas subyacentes a estos procedimientos algorítmicos. Si esto fuera así, uno podría concluir que los conceptos de "verdad lógica" y de "verdad en una interpretación" se manejaban con soltura por entonces. Sin embargo, desde su punto de vista, hablar de "semántica" en este periodo fundacional sería algo anacrónico. En su lugar, introducen el término "protosemántica ${ }^{18 "}$ para referirse a dichos procedimientos algorítmicos.

En consecuencia, si no hay una clara distinción entre la semántica, por un lado, y el cálculo, por otro, ¿en qué sentido podemos hablar de completud, del equilibrio entre cálculos y modelos? En palabras de Manzano y Alonso:

The completeness of propositional logic can be seen, therefore, as a consequence of the decidability of a designated subset of the formulae of the language and not as a way to compare two basic and independent concepts: namely those of proof and truth. (Manzano y Alonso 2014, 7-8)

En este trabajo, esperamos haber mostrado que la completud débil de la lógica proposicional no se obtiene intentando responder a la pregunta de si todas las tautologías son teoremas lógicos (es decir, de si el cálculo es suficiente para generar sin premisas al conjunto de las tautologías). Más bien, es un resultado inmediato que se sigue del hecho de que los axiomas 1-4 son Post completos y de la corrección de los "axiomas materiales" (de las reglas de inferencia). Pues, en efecto, si todas las fórmulas deducibles a partir de los mismos (los teoremas lógicos) son iguales a 0 en la interpretación aritmética (esto es, son fórmulas válidas) y si no hay ninguna fórmula que no siendo un teorema lógico sea igual a 0 en dicha interpretación (Post completud), entonces la clase de los teoremas lógicos coincide con la de fórmulas válidas. ( $c f$. Hilbert 1917-18, 158). De este modo,

\footnotetext{
17 "Ob das Axiomensystem wenigstens in dem Sinne vollständig ist, daß wirklich alle logischen Formeln, die für jeden Individuenbereich richtig sind, daraus abgeleitet werden können, ist eine noch ungelöste Frage" (Hilbert y Ackermann 1928, 869).

18 "To refer to all these mechanisms of classification of formulae based on the attribution of some property that functionally spreads through their structure, we use the term protosemantics. Truth tables and normalform algorithms are part of protosemantics and so are other tabular methods and classification algorithms employed to establish independence results in axiomatic systems" (Manzano y Alonso 2014, 4-5).
}

Revista de Humanidades de Valparaíso, 2019, No 14, 449-466

(c) (1) @ $९$ CC BY-NC-ND 
tendríamos la respuesta a la pregunta por la completud débil del cálculo de enunciados antes de que esta se formulase.

Esto explicaría, de hecho, por qué la completud débil de la lógica proposicional no fue considerado un resultado de especial relevancia. Hilbert $(1917-18,158)$ llega a la conclusión de que el cálculo de enunciados es Post completo en una nota al pie, mientras que Bernays (1918) no menciona este hallazgo en la carta (datada del 8 de abril de 1920) que escribió a Russell con motivo de su descubrimiento de que la proposición primitiva "Assoc" era en realidad deducible a partir de las demás y que el resto sí eran independientes entre sí.

Como hemos visto más arriba, fue necesario darse cuenta de que la lógica de primer orden era Post incompleta para preguntarse por su completud débil. La Post incompletud muestra, en el fondo, que hay fórmulas que no siendo teoremas lógicos sí son iguales a 0 en la interpretación aritmética extendida. Pero el propio Hilbert se dio cuenta de que ser igual a 0 en la interpretación aritmética es ser verdadero en un modelo particular, a saber, uno que consta de un solo elemento. De ahí es inmediato preguntarse si hay fórmulas de la lógica de primer orden que no siendo teoremas lógicos sean verdaderas en toda interpretación, en todo modelo. La respuesta es que no (pues, como hemos dicho, la lógica de primer orden es débilmente completa), pero dar con esa respuesta requeriría un entendimiento más profundo de lo que significan los conceptos de "verdad lógica" y de "verdad bajo una interpretación". Este mayor entendimiento (o sea, el paso de una protosemántica a una semántica) llegaría con Gödel $(1929,1930)$ y con Tarski, pero desborda, sin duda, los límites del presente ensayo.

\section{Conclusiones}

La conclusión principal de este trabajo es que la Post incompletud de los axiomas para la lógica de primer orden posibilitó que la completud débil de la misma fuera vista como un problema a resolver en los Grundzüge der Theoretischen Logik. Esta incompletud muestra que hay fórmulas iguales a 0 en la interpretación aritmética extendida que no son teoremas lógicos. Por tanto, la pregunta de si todas las fórmulas que son verdaderas en cualquier interpretación son teoremas lógicos aflora con naturalidad.

En la lógica proposicional, ser válido equivale a ser igual a 0 en la interpretación aritmética, que es básicamente la semántica estándar de las conectivas. En la escuela de Hilbert, se desarrollaron las formas normales conjuntiva y disyuntiva, que permitían decidir, en un número finito de pasos, si cualquier expresión proposicional era una tautología o si era satisfacible, respectivamente. Estos procedimientos hacen que se dé menos importancia a la presentación de las reglas del cálculo, pues no necesario encontrar una prueba de $\varphi$ a partir de los axiomas 1-4 para saber si $\varphi$ es una tautología o no lo es. Basándose en estos procedimientos, Hilbert (1917-18) ideó una ingeniosa prueba de que los axiomas del 
cálculo de enunciados son Post completos a partir de la cual (y de la corrección) Bernays (1918), en su segunda tesis de habilitación, fue capaz de concluir la completud débil de la lógica proposicional. Aunque la completud débil aparece formulada con mayor claridad en Bernays (1918) que en Hilbert (1917-18), donde solo es una nota a pie de página, sería erróneo pensar - como ya han advertido Manzano y Alonso (2014) — que se obtuvo como un intento de demostrar el equilibrio entre cálculos y modelos.

\section{Agradecimientos}

Este trabajo ha sido posible gracias a la beca FPU15/00830, concedida por el Ministerio de Educación español. Agradezco a Paolo Mancosu que me facilitara una copia de la carta que Bernays escribió a Russell en 1920. Gracias también a los dos revisores anónimos de la Revista de Humanidades de Valparaíso por sus interesantes comentarios y sugerencias.

\section{Referencias bibliográficas}

Bernays, P. (1918). Beiträge zur axiomatischen Behandlung des Logik-Kalküls. En Hilbert, D. (2013), David Hilbert's Lectures on the Foundations of Arithmetic and Logic 1917-1933, W. Ewald y W. Sieg (eds.), pp. 231-269. Berlin: Springer.

Dreben, B., van Heijenoort, J. (1986). Introductory note to 1929, 1930 and 1930a. En Gödel, K. (1986), Collected Works, vol. 1. S. Feferman et al. (eds.), pp. 44-59. Oxford: Oxford University Press.

Gödel, K. (1929). On the completeness of the calculus of logic. En Gödel, K. (1986), Collected Works, vol. 1. S. Feferman et al. (eds.), pp. 61-101. Oxford: Oxford University Press.

Gödel, K. (1930). The completeness of the axioms of the functional calculus of logic. En Gödel, K. (1986), Collected Works, vol. 1. S. Feferman et al. (eds.), pp. 103-123. Oxford: Oxford University Press.

Gödel, K. (1986). Collected Works, vol. 1, S. Feferman et al. (eds.). Oxford: Oxford University Press.

Hilbert, D. (1904). On the foundations of logic and arithmetic. En van Heijenoort, J. (ed.) (1967), From Frege to Gödel. A Source Book in Mathematical Logic 1879-1931, pp. 129-138. Harvard: Harvard University Press.

Hilbert, D. (1917-18). Prinzipien der Mathematik. En Hilbert, D. (2013). David Hilbert's Lectures on the Foundations of Arithmetic and Logic 1917-1933, W. Ewald y W. Sieg (eds.), pp. 59-219. Berlin: Springer.

Hilbert, D. (2013). David Hilbert's Lectures on the Foundations of Arithmetic and Logic 19171933. W. Ewald y W. Sieg (eds.). Berlin: Springer. 


$$
\begin{gathered}
\text { Completud débil y Post completud en la escuela de Hilbert } \\
\text { Víctor Aranda }
\end{gathered}
$$

Hilbert, D., Ackermann, W (1928). Grundzüge der theoretischen Logik. En Hilbert, D., (2013). David Hilbert's Lectures on the Foundations of Arithmetic and Logic 1917-1933, W. Ewald y W. Sieg (eds.), pp. 809-916. Berlin: Springer.

Lewis, C. I. (1918). A Survey of Symbolic Logic. Berkeley y Los Angeles: University of California Press.

Löwenheim, L. (1915). On possibilities in the calculus of relatives. En En van Heijenoort, J. (ed.) (1967), From Frege to Gödel. A Source Book in Mathematical Logic 1879-1931, pp. 228251. Harvard: Harvard University Press.

Mancosu, P. (2010). The Adventure of Reason. Interplay between Philosophy of Mathematics and Mathematical Logic, 1900-1940. Oxford: Oxford University Press.

Manzano, M., Alonso, E. (2014). Completeness: from Gödel to Henkin. History and Philosophy of Logic, 35(1): 1-26. https://doi.org/10.1080/01445340.2013.816555

Monk, R. (1994). Ludwig Wittgenstein. Barcelona: Editorial Anagrama.

Post, E. (1921). Introduction to a general theory of elementary propositions. En van Heijenoort, J. (ed.) (1967), From Frege to Gödel. A Source Book in Mathematical Logic 1879-1931, pp. 264-283. Harvard: Harvard University Press.

van Heijenoort, J. (ed.) (1967). From Frege to Gödel. A Source Book in Mathematical Logic 1879-1931. Harvard: Harvard University Press.

Wittgenstein, L. (1922). Tractatus logico-philosophicus. Londres: Kegan Paul.

Zach, R. (1999). Completeness before Post: Bernays, Hilbert and the development of propositional logic. Bulletin of Symbolic Logic, 5(3): 331-366. 